\title{
Life Threatening
}

National Cancer Institute

\section{Source}

National Cancer Institute. Life Threatening. NCI Thesaurus. Code C82508.

A situation that imperils the existence of an entity. 